\title{
Congenital hepatic fibrosis: the long-term prognosis
}

\author{
D. N. S. KERR ${ }^{1}$, STELLA OKONKWO, AND R. G. CHOA \\ From the Department of Medicine, University of Newcastle upon Tyne, Newcastle upon Tyne
}

SUMMARY The long-term prognosis in congenital hepatic fibrosis has been assessed in 30 patients, 13 treated at Newcastle and 17 from other British centres. Twenty-four patients had been followed-up for more than five years from diagnosis. Shunt operations, performed in 18 patients, were successful in controlling haemorrhage with a low mortality $(1 / 18)$, low incidence of recurrent haemorrhage $(3 / 18)$ and portal-systemic encephalopathy $(3 / 18)$ but with a higher incidence of postoperative jaundice (7/18). About a third of the survivors had some evidence of hepatic dysfunction but none had chronic portal systemic encephalopathy. Intelligence quotient was close to normal but educational achievement and job status were low. Serum albumin fell after shunt surgery and a few patients developed ascites: anaemia and hypersplenism however, were corrected by surgery. Blood ammonia is raised in survivors with congenital hepatic fibrosis and rises further after a normal meal; further observations are needed on cerebral function after several decades of survival.

\section{Personal note (D.N.S.K.)}

When I left Hammersmith in 1959 I was transmuted overnight into a nephrologist and none of my subsequent researches is likely to interest readers of Gut. However, I retained a tenuous link with gastroenterology through an uncommon disease which was first fully described during my stay on the Sherlock unit. My first task there was to investigate two children with portal hypertension; it had been attributed to cirrhosis but there were bizarre features: a strong family history-they were brother and sister and a third sib was affected; very hard liver, which bent the Vim Silverman needle; wellpreserved hepatic function; cystic disease of the kidneys; hepatic histology which showed dense fibrous bands, containing dysplastic bile ducts and sparse portal venules, around almost normal islands of hepatic tissue. Eight such children from Hammersmith were reported to the Association of Physicians (Harrison et al., 1959) and with four others from Newcastle were published under the title 'Congenital hepatic fibrosis' (Kerr et al., 1961), which has stuck. The puzzling variety of renal lesions that can accompany the hepatic disease-and may overshadow it-was described the following year (Kerr et al., 1962); the commonest is renal tubular ectasia (Unite et al., 1973; Six et al., 1975) but nephronophthisis (Boichis et al., 1973; Robins et al., 1976) and a lesion closely resembling adult polycystic disease

${ }^{1}$ Address for reprints: Professor D. N. S. Kerr, Department of Medicine, Royal Victoria Infirmary, Newcastle upon Tyne NE1 4LP.
(Sommerschild et al., 1973) can occur or the kidneys may be normal. The variable renal pathology led Murray-Lyon and his colleagues (1973) to question whether congenital hepatic fibrosis was a single entity but there is sufficient diversity within single families to suggest that it is. However the genetic basis remains obscure. If so uncommon a disease were due to a recessive gene, as I suggested in 1961, one would have expected reports of consanguinity in parents by now. If it were a dominant (Sweetnam and Sykes, 1961) it should have appeared in successive generations. The few studies of karyotype have been normal (Thorburn et al., 1967).

An invitation to contribute to Professor Sherlock's Festschrift has stimulated me to report a study carried out by my co-authors as a student project in 1974. It relied heavily on the co-operation of Professor Sherlock, Dr A. M. Dawson, Dr W. P. Sweetnam, Professor A. E. Read, and Dr R. Williams who helped us to trace surviving British patients and gave us access to their case notes. Dr J. Raffenberger, Dr L. Brandberg, and Dr J. Pirrie sent us details of published cases. Their help is gratefully acknowledged.

Most accounts of congenital hepatic fibrosis stress the well-preserved hepatic function, the good prognosis if bleeding from varices can be controlled, and the suitability of the patients for shunt surgery. However, the published information on long-term survival and rehabilitation is scant. Even in the short term, hepatic failure can occur (Kerr et al., 1961; Williams et al., 1964) and it would not be surprising 
if hepatic function declined after shunt operations. Children with the disorder face other hazards, including uraemia, calculi (Hodgson et al., 1976), and septicaemia (Kerr et al., 1962) from their renal disease, cholangitis from associated anomalies of the bile ducts which include Caroli's anomaly (cystic dilatation) (Murray-Lyon et al., 1972; Mall et al., 1974), choledochous cyst, and gallstones; infections from other portals of entry (Thorburn et al., 1967), and lung fibrosis or emphysema (Williams et al., 1964). Peptic ulcer has featured in some reports (Lorimer et al., 1967) and might be expected to increase after shunt surgery. Sommerschild et al. (1973) reviewed 75 published cases half of which had terminated fatally by the time they were published, mainly from bleeding or renal failure.

Shunt surgery in the adult exacts a heavy penalty in cerebral dysfunction (Hourigan et al., 1971). The problem is smaller if the liver is not cirrhotic (Zeegen et al., 1970a), but the child with congenital hepatic fibrosis is a rather special case. Surgery may be required during the first decade of an expected long life; even if he escapes portal systemic encephalopathy, which is mainly a problem of the middleaged and elderly (Hourigan et al., 1971), he may sustain more subtle brain damage leading to educational retardation. We therefore tried to trace patients with congenital hepatic fibrosis long after presentation to chart their subsequent course.

\section{Methods}

\section{PATIENTS}

We knew, or were told, of 43 British patients with congenital hepatic fibrosis but could find the hospital case notes of only 30 . Since case records are more likely to be preserved if the patient is alive this probably biased our series in favour of survivors. Of four missing records in Newcastle two belonged to children known to have died from recurrent haemorrhage and a third has since been traced; the patient died of gangrene of the jejunum, attributed at necropsy to portal vein thrombosis, eight years after a successful portacaval shunt performed at the age of 12 years. The fourth patient has turned up alive and well, apart from recurrent urinary infection in her medullary sponge kidneys, 18 years after portacaval anastomosis at the age of 18 years. These four are not included in the 30 described below.

Of the 30 whose notes we found, 21 were alive, seven dead, and two lost to follow-up, presumed alive. Two of us (S.O. and R.G.C.) interviewed 15 of these patients; the remaining information was gathered from hospital notes, through the courtesy of those acknowledged above.
ASSESSMENT OF MENTAL FUNCTION

We enquired about the patient's educational achievements, employment, and personal relationships. Intelligence was tested with the Standard Progressive Matrices (a non-verbal test of the ability to see relationships between progressively more difficult geometrical figures) and the Mill Hill Vocabulary Scale. The latter is a verbal test consisting of two sets of $\mathbf{4 0}$ words, each arranged in order of the frequency with which the word appears in everyday language; the patient is asked to write down the meaning of each word in the first set and select a synonym from the second. Two patients under 14 years old were given the junior versions of these tests.

\section{ASSESSMENT OF LIVER FUNCTION}

Laboratory data were extracted from case notes and were therefore performed by various methods in different hospitals. We studied blood ammonia by the method of Horn and Squire (1966). Ammonia is extracted from plasma by absorption on to a column of strong cation exchange resin. This is then eluted with sodium chloride solution and the ammonium concentration estimated by the indophenol blue reaction.

\section{Results}

\section{POPULATION STUDIED}

Histological reports, and liver biopsies when available, were reviewed. Twenty-seven patients had a definite diagnosis of congenital hepatic fibrosis; three were regarded by the reporting pathologist as probable cases and were accepted by us as such. There were 17 females and 13 males aged 7 to 69 years. The average age at presentation was 18 years. Of the survivors, three were aged 5-15 years, nine 16-25 years, six 26-35 years, and two were over 35 years. ${ }^{1}$

The age range was higher than in most previous reports but the clinical picture was conventional. Twenty-one of the 30 patients presented with haematemesis or melaena; other presenting features were anaemia, leucopenia and thrombocytopenia, abdominal enlargement and pain, fever, jaundice, ascites, uraemia and hepatosplenomegaly, found incidentally. At presentation 20 had enlarged liver and spleen, five had splenomegaly only and five hepatomegaly only. Two had jaundice, two spider naevi, and one hepatic precoma, confirming previous evidence that hepatic function is sometimes abnormal in this disease.

${ }^{1} \mathrm{~A}$ table with fuller details of individual cases and an indication of which patients appear in previous publications is available from D.N.S.K. on request. 
SURVIVAL

The outcome in these 30 patients, to 1974 , is shown in Figures 1 and 2. Of those who had shunt operations, patient 26 died of recurrent ascending cholangitis and hepatic failure, three years after surgery; patient 27 died at the age of 14 years from hepatic failure and septicaemia, nine years after surgery; patient 28 died of pulmonary embolism following emergency portacaval anastomosis for bleeding at the age of 54 years; patient 29 died of recurrent ascending cholangitis and hepatic failure aged 22 years, 15 years after portacaval anastomosis. Of those who had no shunt, patient 18 died of haematemesis aged 18 years, patient 20 of septicaemia at 30 years. and patient 21 of ascending cholangitis at 27 years.
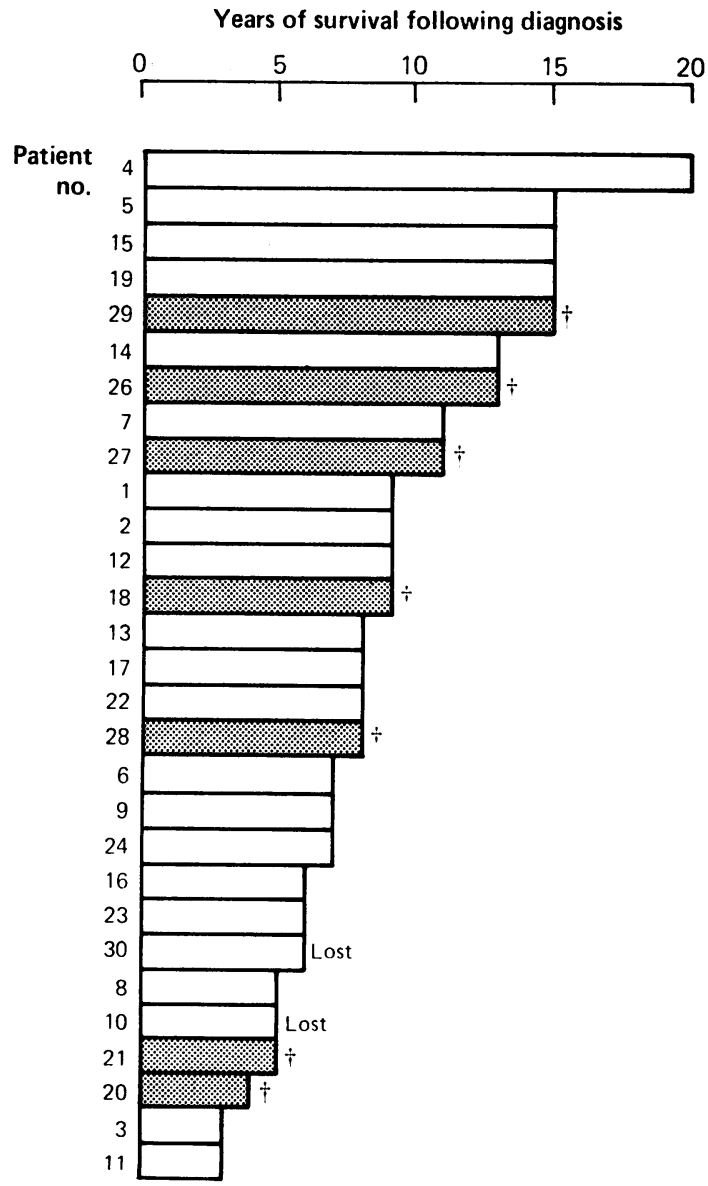

Fig. 1 Duration of survival to follow-up in 1974, or to death, in 30 patients with congenital hepatic fibrosis. Patient No. 25 was followed for 14 years.

SUCCESS OF SHUNT SURGERY AND DEVASCULARISATION

Of the 17 patients who survived the postoperative

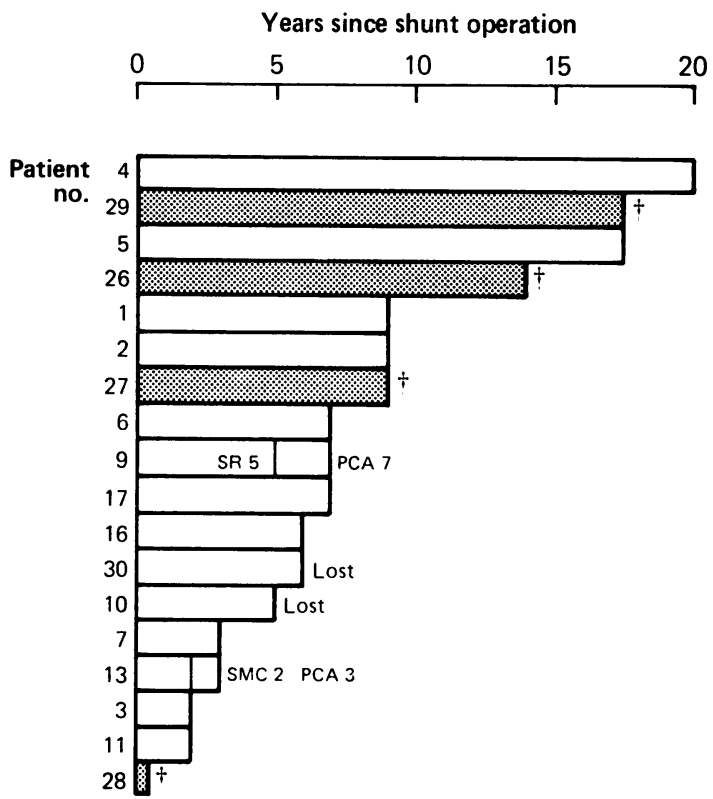

Fig. 2 Duration of survival to follow-up in 1974, or to death in 18 patients who had shunt operations.

period after shunt surgery, two had prophylactic operations and have never bled (patients 1 and 2). Only three of the remainder have had recurrent haemorrhage. Patient 9 bled shortly after a portacaval anastomosis at the age of 12 years, requiring thrombectomy and reanastomosis. The shunt thrombosed again and she bled again one year later but has had no further bleeding following a splenorenal anastomosis. Patient 13 had a further haemorrhage 10 months after portacaval anastomosis but has been well since a superior-mesenteric-caval anastomosis. Patient 3 had recurrent bleeding two months after portacaval anastomosis at the age of 44 years; she was treated conservatively and has had no further trouble.

Four patients had had devascularisation operations. Two had no further bleeding in four years (patients 14 and 15). One had repeated melaena but refused further operation (patient 25) and one died from a massive haematemesis (patient 18).

The 12 who had not undergone surgery for varices were not a control group; most had not bled. One was awaiting surgery for haematemesis and one (patient 12) was treated conservatively for occasional melaena.

\section{NEUROPSYCHIATRIC COMPLICATIONS}

None of the patients had chronic portal-systemic encephalopathy (PSE) either before or after surgery. Patient 18, who had no shunt operation, developed PSE after a massive haematemesis from which he died. Three other patients developed PSE for the 
first time after shunt surgery. Patient 2 had normal liver function tests and electroencephalogram (EEG) before a prophylactic portacaval shunt but had two episodes of PSE at three days and 14 months after surgery; he remained well thereafter on a $40 \mathrm{~g}$ protein diet. Patient 3 developed PSE four years after her portacaval anastomosis and was treated with low protein diet and neomycin; she improved and was able to resume a normal diet. Patient 27 had normal liver function tests until his portacaval anastomosis at the age of 5 years; he had two episodes of PSE at four and nine years after his operation and was maintained at other times on a $\mathbf{4 0} \mathrm{g}$ protein diet and neomycin; he died of hepatic failure and aplastic anaemia four months after his second episode of PSE.

The details of 13 patients who were assessed for intelligence are given in the Table. The mean intelligence of the group on both tests was just below normal, but not significantly so and no patient had a score below 80; about a tenth of the normal population fall below 80 . Patient 1 had IQ tests performed by us before and one year after a shunt operation; full scale Wechsler results were 117, 117\% respectively; there was a slight improvement in the verbal test and a minimal decline in the performance. Patient 4 had had an IQ test at the age of 10 years, when she scored $77 \%$ for her age and had 'slight mental retardation (mental age 9 years)' on the Schonell word list; if anything she had improved when we retested her 12 years later.

Table Intelligence tests in 13 patients with congenital hepatic fibrosis

\begin{tabular}{lllll}
\hline Patient & $\begin{array}{l}\text { Age when } \\
\text { tested }(y r)\end{array}$ & $\begin{array}{l}\text { Years since } \\
\text { shunt } \\
\text { operation }\end{array}$ & \multicolumn{2}{c}{ Percentage points scored in: } \\
\cline { 4 - 5 } & & MHVS* & SPM† \\
\hline 1 & 18 & 6 & $100-110$ & $110-119$ \\
2 & 39 & 7 & 100 & $101-110$ \\
3 & 45 & 1 & $81-90$ & $81-90$ \\
4 & 23 & 18 & $81-90$ & $91-100$ \\
5 & 23 & 12 & $81-90$ & $91-100$ \\
6 & 59 & 5 & $91-100$ & $91-100$ \\
7 & 22 & 1 & $91-100$ & $91-100$ \\
8 & 67 & $n$ & $101-110$ & $91-100$ \\
9 & 17 & 5 & $81-90$ & $81-90$ \\
10 & 21 & 3 & $91-100$ & $91-100$ \\
11 & 14 & 1 & $81-90$ & $81-90$ \\
12 & 12 & n/a & $81-90$ & $91-100$ \\
13 & 10 & 1 & $81-90$ & $91-100$ \\
\hline
\end{tabular}

* Mill Hill Vocabulary Scale.

†Standard Progressive Matrices. Results are scored in 10 mark bands but statistics were performed on raw marks: five of the eight in the 91-100 band for SPM actually scored 100 . Patients 14 and 15 failed to complete their tests.

We sought evidence of constructional apraxia by asking patients to draw a five-pointed star and scored the result as described by Zeegen and col- leagues (1970b). One of eight patients tested had a score at the upper limit of normal (five), the other seven had good normal scores. As a more exacting test of concentration, spatial orientation, and constructional ability, we used the modified Reitan Trail Test which is a slightly more complicated version of the child's dot-to-dot puzzle; the time to completion of two puzzles is the measure of performance. Three out of six patients with shunts, two out of seven without shunts, and two out of six controls had abnormally long times. In summary, we found little evidence of the constructional apraxia which is said to characterise early PSE.

OTHER COMPLICATIONS DURING FOLLOW-UP Three patients developed ascites, one of whom (patient 6) had generalised oedema, probably due to renal failure. Other complications encountered were iron deficiency anaemia, unexplained upper abdominal pain, recurrent urinary infection, hypoglycaemia, dysphagia due to oesophageal transection and the fatal complications already listed. Two patients required cholecystectomy and a third had a liver cyst removed.

\section{CHANGES IN LIVER FUNCTION TESTS}

Twenty-four of our 30 patients had a raised serum alkaline phosphatase at presentation and 26 out of 30 at the end of follow-up, confirming our previous observation that this is the one characteristic abnormality in congenital hepatic fibrosis (Kerr et al., 1961). Only one of 27 patients tested had a raised SGOT (AST) at presentation but eight of 28 tested had a raised level at the end of follow-up. The prothrombin time was below $80 \%$ at presentation in 11 of 27 tested and at the end of follow-up in nine of 27 tested; there was no consistent change after surgery. Serum bilirubin was normal in 27 of 29 patients at presentation, when the highest reading was $4.0 \mathrm{mg} / \mathrm{dl}$ but at the end of follow-up 11 of 30 patients were jaundiced, eight mildly $(1 \cdot 6-4 \cdot 0 \mathrm{mg} / \mathrm{dl})$ and three deeply $(14 \cdot 9-34.0 \mathrm{mg} / \mathrm{dl})$; seven of 18 patients had painless jaundice following shunt surgery. Serum albumin was above $30 \mathrm{~g} / 1$ in 27 of 29 patients at presentation; it fell following shunt surgery (Fig. 3) and remained low during follow-up but there was no consistent change in patients who did not undergo shunt operations (Fig. 4). These findings confirm our previous impression that biochemical evidence of hepatic failure is uncommon at presentation but increases with length of follow-up, particularly after surgery.

Fasting blood ammonia, and the rise after a normal meal, were measured in seven patients with congenital hepatic fibrosis, five with cirrhosis, one with amyloid liver, and seven healthy controls 


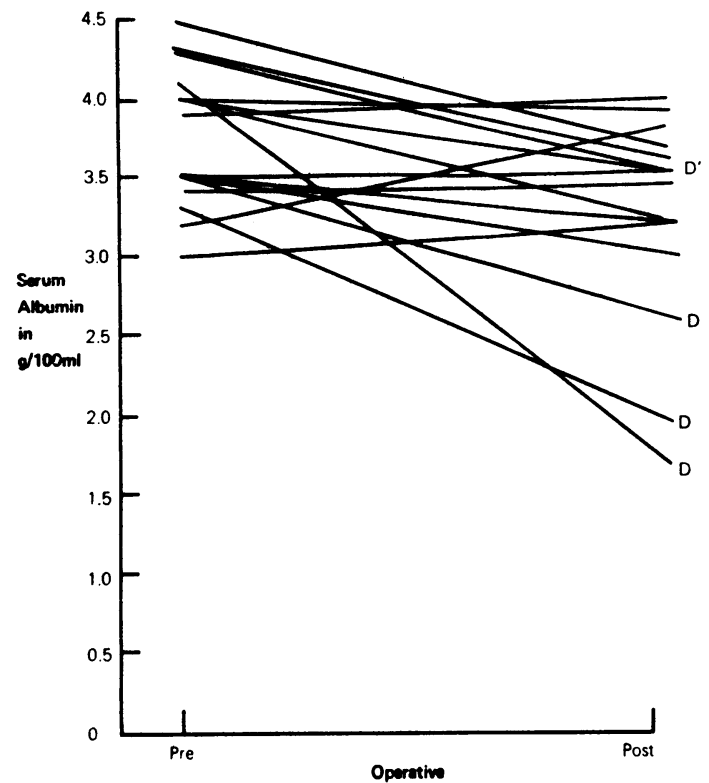

Fig. 3 Changes in serum albumin across the period of shunt surgery in 16 patients. $D=$ died. $($ SI units: $: \times 10 \cdot 0)$.

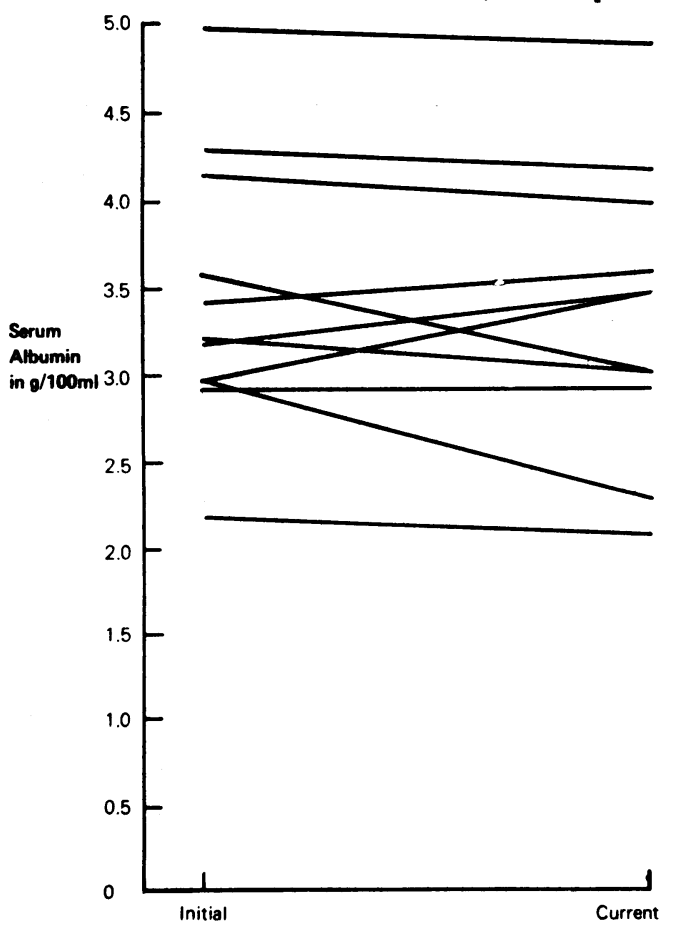

Fig. 4 Change in serum albumin between presentation and follow-up in patients who did not undergo shunt surgery. $($ SI units: $\times 10 \cdot 0)$.

(medical students and laboratory staff). The results in congenital hepatic fibrosis were distinctly abnor-

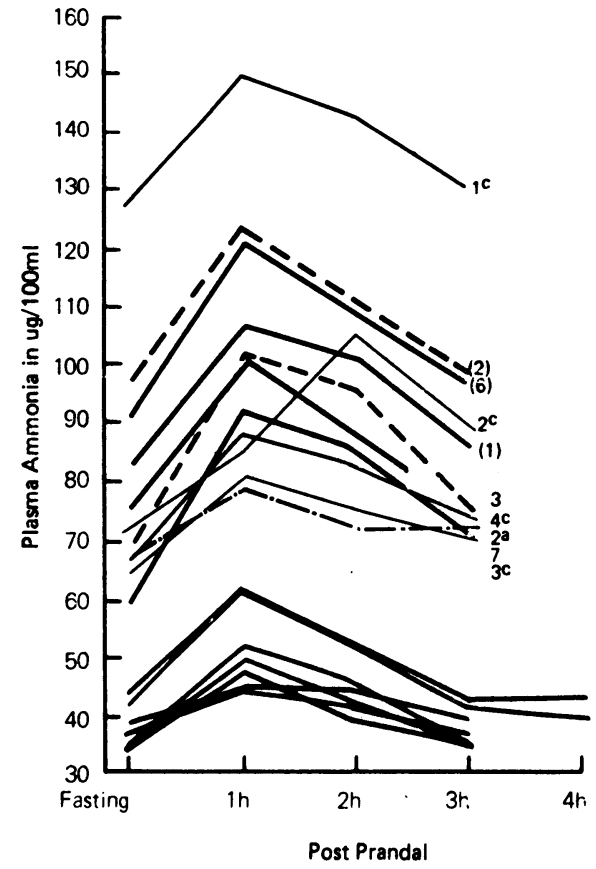

Fig. 5 Blood ammonia, fasting and after a normal meal, in seven patients with congenital hepatic fibrosis, five with other liver diseases, and seven normal controls.

$1-4 c$ cirrhotics. $=1-7 \mathrm{CHF}$ patients.

- Controls. - $-2^{a}$ patient with amyloid liver. (SI units: $\times 10 \cdot 0)$

mal and comparable with those in other liver diseases Fig. 5).

\section{ANAEMIA}

There was a consistent rise in haemoglobin after shunt surgery (Fig. 6) which did not occur in patients not requiring surgery, who were less anaemic (Fig. $7)$. Ten patients had leucopenia $(<4000 / \mathrm{cu} . \mathrm{mm})$ or/and thrombocytopenia ( $<150000 / \mathrm{cu} . \mathrm{mm})$ which was corrected by portacaval shunt or splenectomy.

\section{GENERAL REHABILITATION}

Four of the 23 survivors were still at school, in ordinary schools and apparently performing satisfactorily. The remaining 19 had all been educated at normal schools, although one, who had her portacaval anastomosis at the age of 6 years was regarded as backward and took no competitive tests at school. Her brother who also had a portacaval anastomosis in early childhood, was classified as backward during life. Only two of the remaining 18 survivors had taken any school leaving qualifications (GCE or RSA); they were holding down jobs as laboratory technician and secretary respectively, with success, 


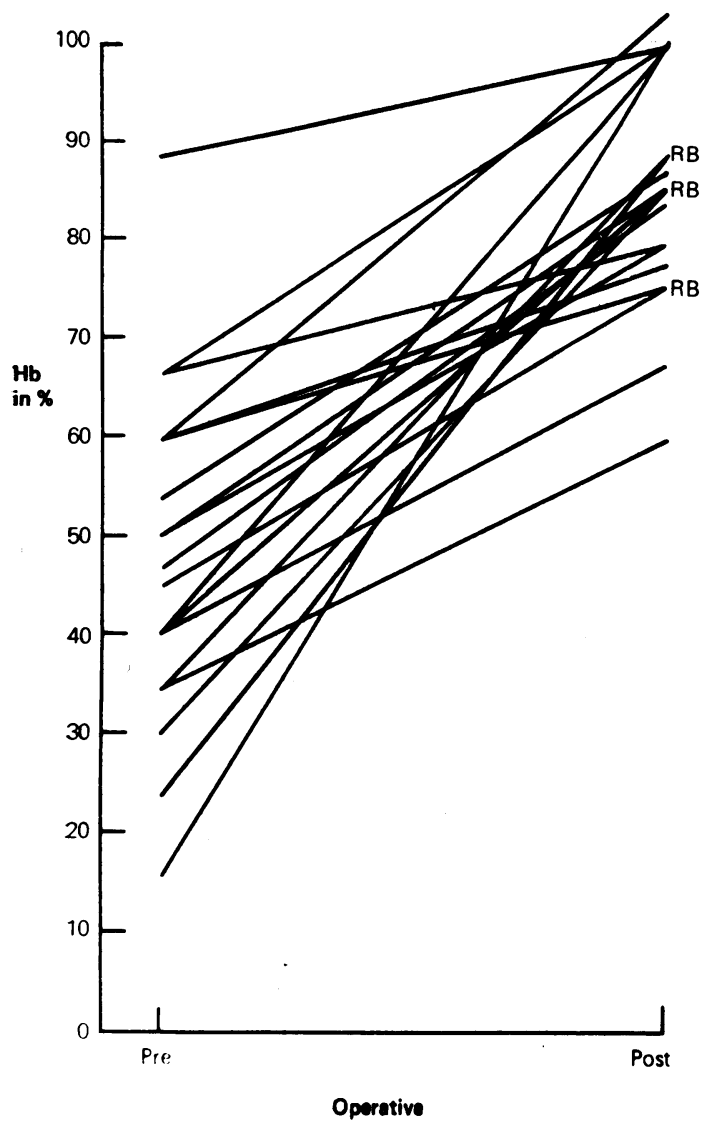

Fig. 6 Changes in haemoglobin across the period of shunt surgery in 18 patients (one of whom had two shunt operations and appears twice). $R B=$ re-bled.

though one had occasional behavioural disturbance. Most of the patients had left school at 14 or 15 years and were in occupations without high intellectual demands.

\section{Discussion}

Thirteen of the 30 patients, plus the four mentioned in the introduction, come from the Northern region, population 3 million. None of the families has more than two affected members so it is likely that the Northern region is representative of the whole country, suggesting that there should be about 300 known patients in the United Kingdom, perhaps half of them still alive. Clearly we failed to locate more than a fraction of the patients in other regions but our collected series is much the largest published and sufficient to give some idea of the long-term prognosis in this disease.

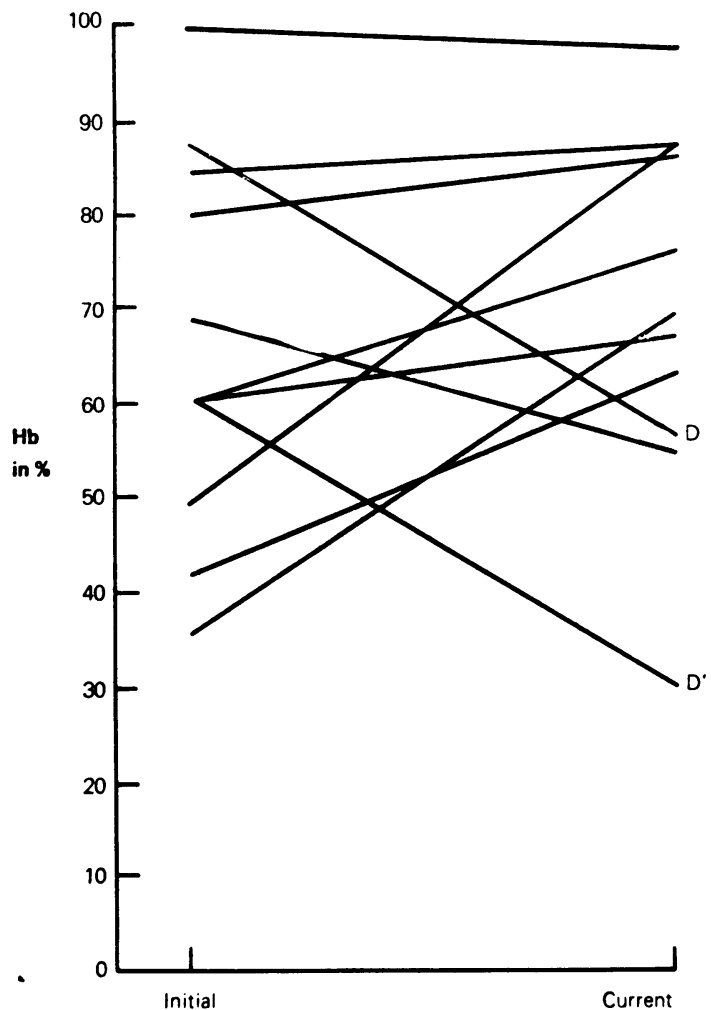

Fig. 7 Changes in haemoglobin between presentation and follow-up in patients who did not undergo shunt surgery.

By and large the impressions gained by 1961 have been supported. Shunt surgery gives good results in control of bleeding and correction of hypersplenism. Operative mortality is acceptably low, though about a quarter of the operated patients will develop jaundice and about one in 10 will have an episode of PSE. Eventual IQ in adult life is little different from normal but educational achievement is poor and eventual job status rather low. It seems likely that this reflects protracted absence from school and inadequate educational support in hospital one to two decades ago, rather than the effects of shunt surgery. Nonetheless, the protracted exposure to high blood ammonia (and presumably other toxins) that we have demonstrated, and the modest incidence of hepatic dysfunction with the passage of time, call for a guarded prognosis for very long-term cerebral function.

\section{References}

Boichis, H., Passwell, J., David, R., and Miller, H. (1973) Congenital hepatic fibrosis and nephronophthisis. A 
family study. Quarterly Journal of Medicine, 42, 221-233. Harrison, C. V., Kerr, D. N. S., Milnes Walker, R., and Sherlock, S. P. V. (1959). Congenital hepatic fibrosis. Quarterly Journal of Medicine, 28, 588. (Abstract.)

Hodgson, H. J. F., Davies, D. R., and Thompson, R. P. H. (1976). Congenital hepatic fibrosis. Journal of Clinical Pathology, 29, 11-16.

Horn, D. B., and Squire, C. R. (1966). The estimation of ammonia using the indophenol blue reaction. Clinica Chimica Acta, 14, 185-194.

Hourigan, K., Sherlock, S., George, P., and Mindel, S. (1971). Elective end-to-side portacaval shunt; results in 64 cases. British Medical Journal, 4, 473-477.

Kerr, D. N. S., Harrison, C. V., Sherlock, S., and Milnes Walker, R. (1961). Congenital hepatic fibrosis. Quarterly Journal of Medicine, 30, 91-117.

Kerr, D. N. S., Warrick, C. K., and Hart-Mercer, J. (1962). A lesion resembling medullary sponge kidney in patients with congenital hepatic fibrosis. Clinical Radiology, 13, 85-91.

Lorimer, A. R., McGee, J., and McAlpine, S. G. (1967). Congenital hepatic fibrosis. Postgraduate Medical Journal, 43, 770-773.

Mall, J. C., Ghahremani, G. G., and Boyer, J. L. (1974). Caroli's disease associated with congenital hepatic fibrosis and renal tubular ectasia. Gastroenterology, 66, 1029-1035.

Murray-Lyon, I. M., Shilkin K. B., Laws, J. W., Illing, R. C., and Williams, R. (1972). Non-obstructive dilatation of the intrahepatic biliary tree with cholangitis. Quarterly Journal of Medicine, 41, 477-489.

Murray-Lyon, I. M., Ockenden, B. G., and Williams, R. (1973). Congenital hepatic fibrosis-is it a single clinical entity? Gastroenterology, 64, 653-656.

Robins, D. G., French, T. A., and Chakera, T. M. H. (1976). Juvenile nephronophthisis associated with skeletal abnormalities and hepatic fibrosis. Archives of Disease in Childhood, 51, 799-801.

Six, R., Oliphant, M., and Grossman, H. (1975). A spectrum of renal tubular ectasia and hepatic fibrosis. Radiology, 117, 117-122.

Sommerschild, H. C., Langmark, F., and Maurseth, K. (1973). Congenital hepatic fibrosis: report of two new cases and review of the literature. Surgery, 73, 53-58.

Sweetnam, W. P., and Sykes, C. G. W. (1961). Congenital fibrosis of the liver as a familial defect. Lancet, 1, 374-376.

Thorburn, M. J., Miller, C. G., and Bras, G. (1967). Congenita lhepatic fibrosis in Jamaican children. Archives of Disease in Childhood, 42, 379-388.

Unite, I., Maitem, A., Bagnasco, F. M., and Irwin, G. A. L. (1973). Congenital hepatic fibrosis associated with renal tubular ectasia. A report of three cases. Radiology, 109, 565-570.

Williams, R., Scheuer, P. J., and Heard, B. E. (1964). Congenital hepatic fibrosis with an unusual pulmonary lesion. Journal of Clinical Pathology, 17, 135-142.

Zeegen, R., Drinkwater, J. E., and Dawson, A. M. (1970b). Method for measuring cerebral dysfunction in patients with liver disease. British Medical Journal, 2, 633-636.

Zeegen, R., Stansfeld, A. G., Dawson, A. M., and Hunt, A. H. (1970a). Prolonged survival after portal decompression of patients with non-cirrhotic intrahepatic portal hypertension. Gut, 11, 610-617. 